See discussions, stats, and author profiles for this publication at: https://www.researchgate.net/publication/48270077

\title{
Le changement climatique - Histoire scientifique et politique, scénarios
} futurs.

Article in La Météorologie · January 2011

DOI: $10.4267 / 2042 / 36295 \cdot$ Source: OA

CITATIONS

2 authors, including:

Jean Jouzel

Laboratoire des Sciences du Climat et l'Environnement

526 PUBLICATIONS 61,637 CITATIONS

SEE PROFILE

\section{READS}

5,726

Some of the authors of this publication are also working on these related projects:

Project $\quad$ Paleoclimatology View project

Project Impact of climate change on water and carbon cycles of melting permafrost of Western Siberia View project 


\title{
Le changement climatique
}

\author{
Histoire scientifique et politique, \\ scénarios futurs
}

\author{
Gérard Mégie ${ }^{(1)}$ et Jean Jouzel ${ }^{(2)}$ \\ (1) Institut Pierre-Simon-Laplace - Service d'aéronomie \\ CNRS et université Pierre-et-Marie-Curie \\ 4, place Jussieu - 75252 Paris Cedex 05 \\ gerard.megie@cnrs-dir.fr \\ (2) Institut Pierre-Simon-Laplace - Laboratoire des sciences du climat \\ et de l'environnement \\ Commissariat à l'énergie atomique et CNRS, Gif-sur-Yvette
}

\section{Résumé}

Cet article retrace l'historique de la prise de conscience scientifique et politique de l'impact des activités humaines sur le climat de la Terre, via l'effet de serre additionnel. Puis il dresse l'état des connaissances scientifiques actuelles sur le réchauffement du climat, en examinant les divers scénarios d'émissions envisageables pour le futur. La fin de l'article est consacrée aux conséquences du changement climatique et au protocole de Kyoto pour la réduction des émissions de gaz à effet de serre.

\section{Abstract}

\section{Global warming: scientific and political history, scenarios for the future}

This paper traces the history of the scientific and political awareness of the impact of human activities on Earth's climate through additional greenhouse effect. Then it gives the present state of scientific knowledge on global warming, looking at the different conceivable emission scenarios for the future. The end of the article deals with the consequences of global warming and with the Kyoto protocol for the reduction of greenhouse gases emissions.

\section{Activités humaines et réchauffement du climat : la prise de conscience}

\section{Le précurseur}

Dès le XIX $\mathrm{X}^{\mathrm{e}}$ siècle, le Suédois Svante Arrhenius attire l'attention sur le fait que l'homme est en train de modifier la composition de l'atmosphère en dioxyde de carbone à travers l'utilisation du charbon. À partir d'un calcul relativement simple, il estime que notre planète devrait se réchauffer de $5{ }^{\circ} \mathrm{C}$ d'ici à la fin du $X^{\mathrm{e}}$ siècle... Mais ce n'est qu'à partir des années 1970 que ce problème de l'action potentielle des activités humaines sur le climat devient l'objet de l'attention des scientifiques.

\section{Les modélisateurs}

Les modélisateurs en prennent d'abord conscience. Les années après la seconde guerre mondiale voient l'apparition des premiers calculateurs, alors bien rudi- mentaires. La prévision du temps est l'un des domaines explorés, à partir de modèles fondés sur un système d'équations physiques qui permettent de décrire le mouvement de l'atmosphère et le cycle de l'eau depuis l'évaporation à la surface des océans jusqu'à la formation des précipitations. Développés en vue d'applications en météorologie, science qui s'intéresse à la prévision des perturbations dont on ne peut suivre l'évolution individuelle que sur quelques jours, ces modèles sont alors utilisés sur des échelles climatiques, celle du mois et audelà. Dans ce cas, les prévisions portent sur des valeurs moyennes et non plus sur le suivi de perturbations individuelles. Les premières expériences conduites dans les années 1960 permettent de vérifier que ces modèles sont aptes à simuler les grandes caractéristiques du climat.

\section{Les climatologues}

Mais les climatologues s'intéressent très vite à la capacité de ces modèles à rendre compte de climats différents de celui dans lequel nous vivons actuellement. Les expériences réalisées au cours des années 1970 examinent le cas du doublement de la teneur en dioxyde de

Ndlr : Cet article est extrait d'un article original intitulé « Les changements climatiques », rédigé par Gérard Mégie et Jean Jouzel et publié dans l'ouvrage collectif Johannesburg 2002. Quels enjeux ? Quelle contribution des scientifiques ? édité par le ministère des Affaires étrangères (Barbault et al., 2002). L'article extrait a également dé jà été publié dans Lettre pigb-pmrc-France $\mathrm{n}^{\circ}$ 14, octobre 2002. Nous remercions le ministère des Affaires étrangères et la rédaction de Lettre pigb-pmrc-France de nous avoir autorisés à le reproduire dans La Météorologie. 
carbone. Elles confirment qu'une telle modification se traduirait par un réchauffement significatif. Ces résultats sont pris au sérieux et motivent réunions scientifiques et rapports d'experts, dont l'un publié en 1979 sur l'initiative de la NSF (National Science Foundation, États-Unis) et aux conclusions déjà très bien documentées. Mais c'est au cours de la décennie suivante que s'opère la véritable prise de conscience, non seulement au sein de la communauté scientifique, mais bien au-delà, à mesure que l'ampleur des conséquences de ce changement climatique, tant écologiques qu'économiques, commence à être cernée.

Sur les aspects scientifiques, les résultats des modèles climatiques s'accumulent. Certes, ils diffèrent sensiblement d'un modèle à l'autre ; ainsi, le réchauffement prévu par quatre modèles différents (trois modèles américains, un anglais) en cas de doublement instantané de la teneur en dioxyde de carbone varie entre 1,5 et $4,5^{\circ} \mathrm{C}$. Cette amplitude d'un facteur 3 dans la valeur de ce que les climatologues appellent dans leur jargon la sensibilité du climat résulte pour l'essentiel de la façon dont est traitée la formation des nuages. Leurs propriétés optiques font qu'à la fois ils absorbent et réfléchissent le rayonnement solaire. Ils sont en outre affectés de façon différente selon qu'il s'agit de nuages hauts ou de nuages bas. Cette complexité fait que le comportement des systèmes nuageux est difficile à prendre en compte dans les modèles et qu'il reste d'ailleurs une source majeure d'incertitude vis-à-vis de la prévision du climat du futur. Mais, au-delà de ces incertitudes, tous les modèles - cette affirmation reste quasi vérifiée maintenant que le nombre de simulations a été multiplié par près d'un facteur 10 - prévoient un réchauffement. Qui plus est, ce réchauffement est systématiquement plus important que celui légèrement supérieur à $1{ }^{\circ} \mathrm{C}$-qui serait obtenu dans le cas d'un doublement de la teneur en dioxyde de carbone (qui correspond à un forçage radiatif de $4 \mathrm{~W} \cdot \mathrm{m}^{-2}$ ) en l'absence de toute rétroaction climatique.

Il y a donc des mécanismes amplificateurs qui dominent la réponse du climat lors d'un accroissement de l'effet de serre, et cela de façon tout à fait démontrable. Ainsi, un réchauffement de l'atmosphère va, avec un certain délai, se transmettre aux couches de surface de l'océan. Cela entraîne une augmentation de l'évaporation (qui croît exponentiellement en fonction de la température) et, par voie de consé- quence, de la quantité de vapeur d'eau présente dans l'atmosphère. Comme la vapeur d'eau est elle-même un gaz à effet de serre, le forçage radiatif est amplifié. La diminution de la glace de mer, très réfléchissante vis-à-vis du rayonnement solaire, en réponse à ce réchauffement progressif de l'océan, constitue un deuxième facteur d'amplification (elle est alors remplacée par une surface océanique beaucoup plus absorbante). À l'inverse, aucun des mécanismes compensateurs qui peuvent être évoqués n'apparaît efficace.

\section{La communauté scientifique}

Dans une large majorité, la communauté scientifique est rapidement convaincue de l'ampleur du problème et de la nécessité d'en analyser toutes les facettes. Les premiers résultats obtenus sur les glaces antarctiques de Vostok suggèrent également que le climat est un système amplificateur et participent de cette prise de conscience. À l'évidence, celle-ci bénéficie également du fait que les températures moyennes mesurées à la surface de la Terre dans les années 1980 sont clairement à la hausse... La communauté s'organise alors en conséquence. Dès 1979, l'Organisation météorologique mondiale (OMM) met sur les rails le Programme mondial de recherche sur le climat (PMRC). Puis le CIUS, comité international qui regroupe l'ensemble des organisations scientifiques, conscient des interactions fortes qui existent entre climat et environnement, lance en 1986 un programme ambitieux dédié à l'étude de la géosphère et de la biosphère, le Programme international géosphère-biosphère (PIGB). PMRC et PIGB sont deux des quatre composantes de ce qui est désormais connu sous le nom de Programme Global Change (les deux autres sont consacrées à la biodiversité et à la dimension humaine du changement climatique). Grâce à ces initiatives internationales, bien relayées dans de nombreux pays par les organismes de recherche, la compréhension des mécanismes complexes qui gouvernent l'évolution du climat a largement progressé au cours des vingt dernières années.

\section{Les gouvernements}

Les gouvernements ne tardent d'ailleurs pas trop à emboîter le pas, conscients qu'il s'agit là d'un problème que l'on ne peut délibérément ignorer. D'autant que le protocole qui bannit la production de composés contribuant à la destruction de la couche d'ozone, signé à Montréal en 1987, constitue un premier exemple de ce qui peut être entrepris en matière d'environnement planétaire. Avec le recul, les décisions prises dans ce cadre s'avèrent tout à fait judicieuses et, grâce à elles, on peut espérer un ralentissement de l'altération de la couche d'ozone dans les décennies à venir, puis une inversion du processus. Le consensus scientifique qui s'est rapidement établi, les conséquences bien identifiées et le nombre restreint de producteurs ont largement facilité la signature, puis la mise en œuvre de ce protocole de Montréal. Mais, en dépit de nombreuses similarités entre les deux questions, la réponse apportée au cas de l'ozone stratosphérique n'est pas entièrement extrapolable au problème du changement climatique, dont la complexité est beaucoup plus grande. L'existence d'un lien entre activités humaines et réchauffement climatique ne repose alors que sur les prévisions des modèles, dont on sait qu'ils restent assez rudimentaires, et les conséquences de ce réchauffement sont mal cernées. Par ailleurs, si des mesures visant à limiter l'utilisation des combustibles fossiles doivent être décidées, elles seront difficiles à faire accepter tant les notions de développement économique et de confort individuel sont étroitement mêlées à celle de consommation d'énergie. La première étape est donc d'établir un diagnostic.

\section{La création du Giec}

C'est dans ce but qu'en 1988 se crée, sous les auspices conjoints du Programme des Nations unies pour l'environnement (PNUE) et de l'OMM, le Groupe intergouvernemental d'experts sur l'évolution du climat (Giec), aussi connu sous son sigle anglais d'IPCC (Intergovernmental Panel on Climate Change). Le problème du réchauffement du climat est alors pris en compte sur un plan plus politique et constitue l'un des thèmes centraux abordés lors du Sommet de la Terre de Rio, en 1992, au cours duquel est discutée la Convention cadre des Nations unies sur le changement climatique (CCNUCC), ratifiée en 1994. En réponse aux arguments très documentés apportés par les scientifiques à travers les travaux du Giec, le problème est rapidement pris au sérieux par les gouvernements. La CCNUCC s'organise autour des conférences des parties, qui, de Berlin en 1995 à Kyoto en 1997, puis à Marrakech en 2001, ont posé de nombreux jalons visant à 


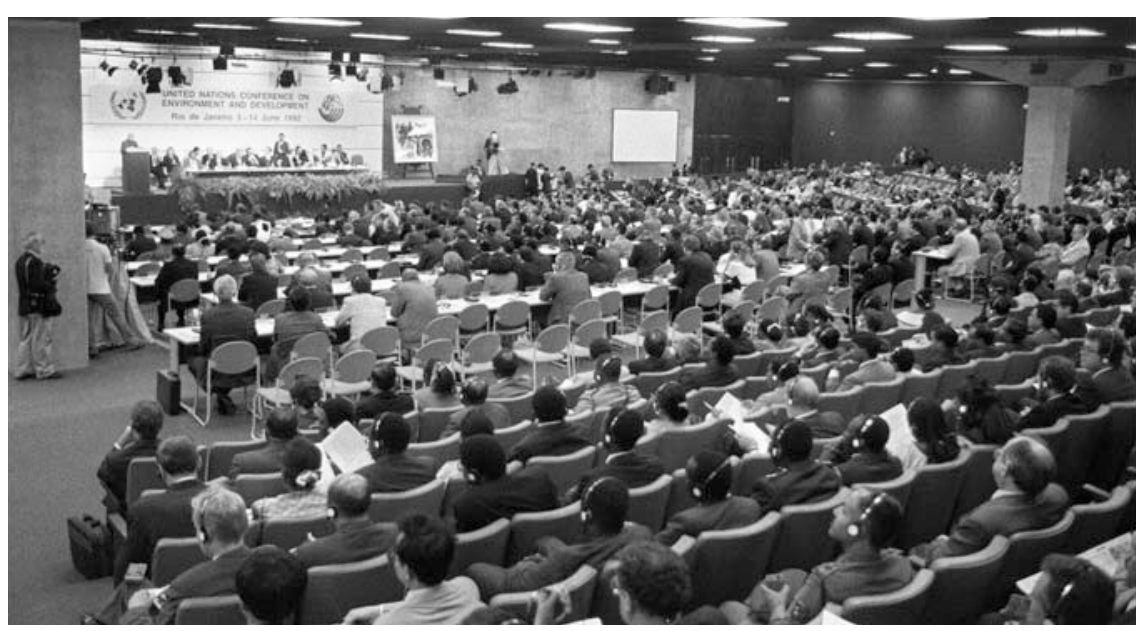

Session d'ouverture du Sommet de la Terre, à Rio de Janeiro (Brésil), le 12 juin 1992. (Photo AFP, Daniel Garcia)

mettre en place des règles de limitation des émissions anthropiques de gaz à effet de serre.

\section{De Rio à Marrakech : l'empreinte des rapports du Giec}

Dès le départ, le Giec s'intéresse à trois volets distincts : le groupe I traite des aspects scientifiques de l'évolution du climat, les deux autres étant respectivement en charge d'examiner les impacts socio-économiques et les mesures possibles d'adaptation (groupe II) et d'étudier les possibilités de limitation des émissions et d'atténuation du changement climatique (groupe III). À ce jour,

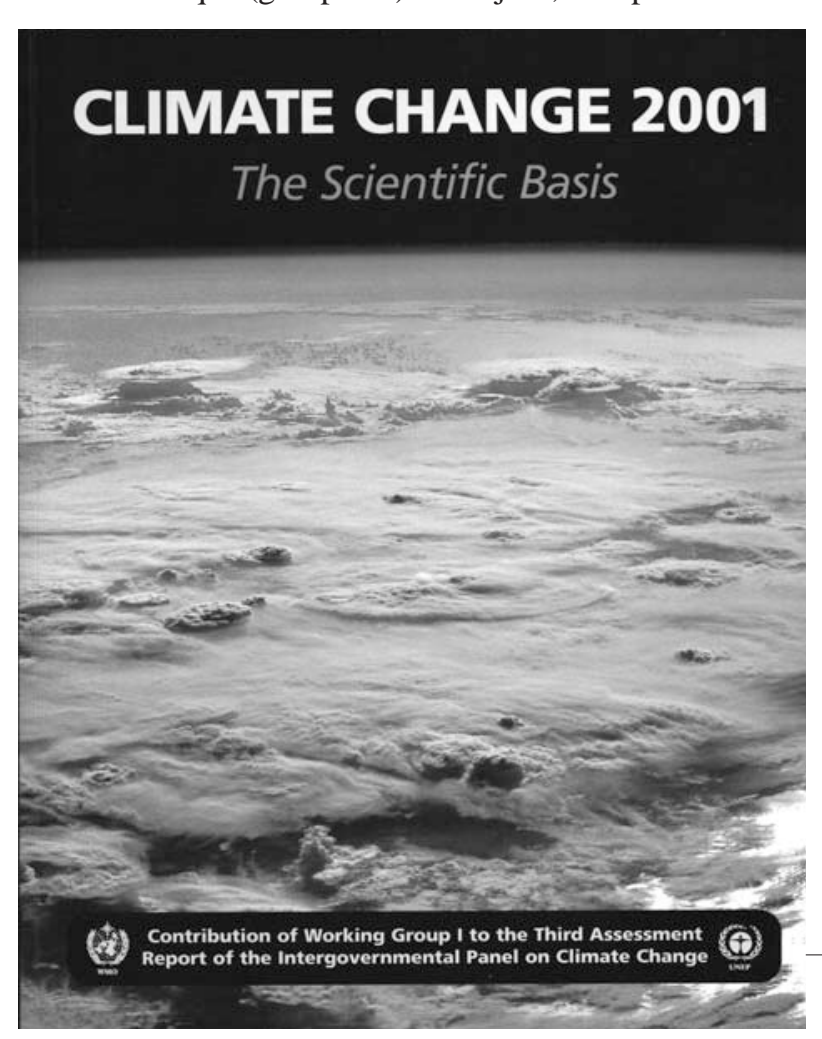

trois rapports complets ont été publiés, le premier en 1990, le deuxième en 1996 et le troisième en 2001. Chaque rapport individuel est divisé en chapitres, dont la première rédaction est confiée à une équipe d'une dizaine de chercheurs de différents pays. Pour accomplir cette tâche, chaque rédacteur sollicite des contributions de chercheurs impliqués dans le domaine concerné. À partir de ces très volumineux rapports (près d'un millier de pages), sont rédigés des résumés d'une cinquantaine de pages, puis des « résumés pour décideurs » beaucoup plus courts et écrits de façon très accessible. Le tout est complété d'un rapport de synthèse. Une fois rédigé, chacun de ces documents reçoit les commentaires de la communauté scientifique (relecteurs) et ceux des représentants des instances gouvernementales. Le processus de rédaction et de relecture prend, à chaque fois, plus de deux ans avant que soit proposé aux gouvernements un texte qui ait l'approbation de la communauté scientifique. Le chiffre de 3000 intervenants, mentionné pour le deuxième rapport, comprend les rédacteurs (une centaine), les contributeurs (quelques centaines) et les relecteurs. Les commentaires provenant des différentes sources (communauté scientifique, instances gouvernementales, mais

Couverture du rapport 2001 du groupe I du Giec. aussi organisations non-gouvernementales) sont pris en compte par les rédacteurs et les textes sont amendés en conséquence. Si les rédacteurs jugent un commentaire non recevable, ce qui est parfois le cas, ils doivent justifier de la ou des raisons qui les ont conduits à ne pas le prendre en considération.

\section{Un consensus entre plus de cent pays}

Vient alors la dernière étape avant publication, celle de l'approbation par les gouvernements membres du Giec (plus d'une centaine de pays). Les « résumés pour décideurs » sont discutés ligne à ligne par les délégués de ces différents pays et approuvés, après des modifications éventuelles, au cours de réunions auxquelles peuvent assister, à titre d'observateurs, des représentants d'organisations non-gouvernementales. La règle est celle du consensus et celuici est quelquefois fort difficile à atteindre malgré les efforts des responsables du Giec et des rédacteurs présents à cette réunion finale, dont l'objectif commun est de parvenir à une solution acceptable par tous et qui reflète bien les rapports complets. Le contenu de ces derniers n'est pas rediscuté, mais celui des résumés étendus est également soumis à approbation et la cohérence entre les différents étages des rapports fait l'objet d'une très grande attention. Nous nous intéresserons ici essentiellement aux conclusions du groupe I, qui traite de la science du changement climatique. Nous en résumons les travaux à travers une série de quatre conclusions.

\section{La modification de l'atmosphère par les activités humaines et le réchauffement du $X X^{e}$ siècle}

Les deux premières conclusions font l'objet d'un consensus qui s'est affirmé au fil des trois rapports du Giec. L'une, que nous avons déjà largement traitée, affirme que les activités humaines modifient la composition de l'atmosphère en gaz à effet de serre. L'autre, déjà évoquée, concerne le réchauffement observé au cours du $\mathrm{XX}^{\mathrm{e}}$ siècle (figure 1). Déjà visible au moment du premier rapport du Giec, l'enregistrement disponible jusqu'à l'année 2001 en donne désormais une idée plus claire. 


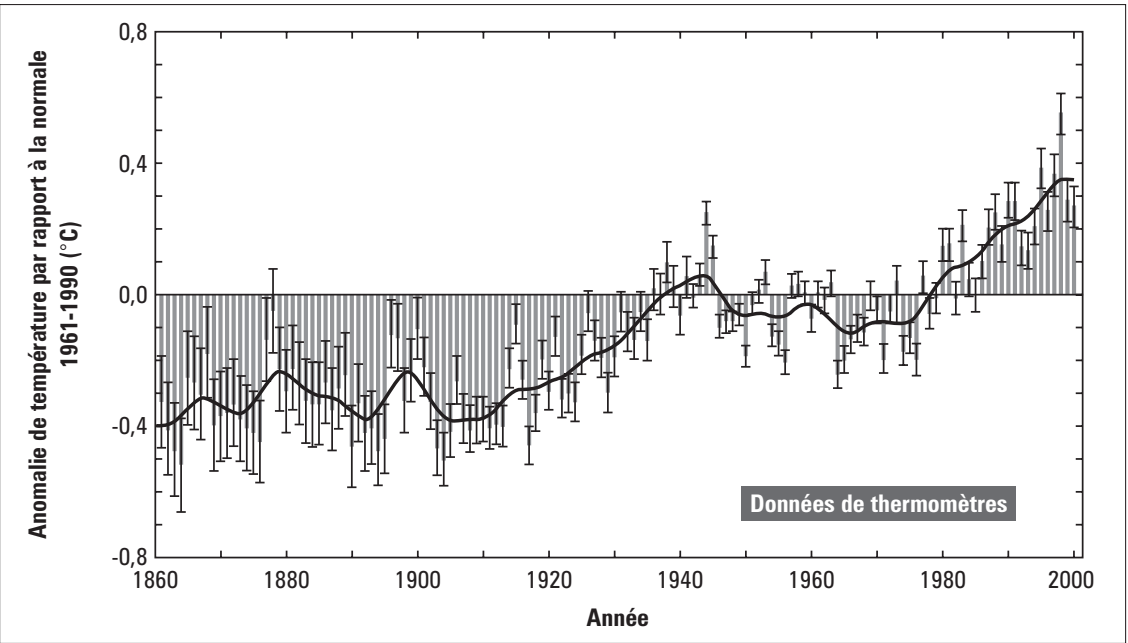

Figure 1 - Variation de la température moyenne de la planète depuis 1861. (Adaptée du rapport IPCC 2001, [www.grida.no/climate/ipcc tar/wg1/index.htm])

Ce réchauffement s'est accéléré au cours des années récentes : l'année 1998 a été la plus chaude depuis 1880 et, si l'on cherche les dix années les plus chaudes, elles sont concentrées au cours des deux dernières décennies. C'est ce graphique pris dans son ensemble qui permet aux experts de conclure que notre climat s'est réchauffé d'un peu plus d'un demi-degré $\left(0,6{ }^{\circ} \mathrm{C}\right.$ avec une incertitude de $\left.\pm 0,2{ }^{\circ} \mathrm{C}\right)$ depuis la fin du XIX ${ }^{\mathrm{e}}$ siècle. Ce réchauffement s'est opéré en deux étapes : la première entre 1910 et 1945 , la seconde depuis 1976. Certains aspects - qualité et couverture géographique des données les plus anciennes, corrections qu'il faut apporter aux températures océaniques mesurées par les bateaux marchands, biais apporté par l'urbanisation pour les stations qui, initialement à la campagne, se sont peu à peu retrouvées en milieu urbain... - ont fait l'objet de nombreuses discussions. Ces points ont été pris en compte de façon indépendante par différentes équipes et les courbes obtenues sont tout à fait similaires.

À noter aussi la polémique qui s'est engagée lorsque l'on a comparé, sur leur période de recouvrement, les températures mesurées à la surface de la planète et celles obtenues à partir de ballons-sondes et d'observations satellitaires. La température de l'atmosphère observée depuis 1979 à l'aide des deux dernières méthodes augmente trois fois moins vite que celle de la surface et il est difficile de dire s'il s'agit là d'un phénomène réel ou si cette différence est liée au fait que la période de comparaison est relativement courte. Par contre, de nombreuses observations témoignent de façon indirecte de ce réchauffement : l'étendue des glaciers alpins diminue de façon quasi générale (les exceptions s'expliquent, soit par la modification de la circulation atmosphérique, soit par l'augmentation des précipi- tations), la couverture neigeuse et l'épaisseur de la glace de mer décroissent dans beaucoup de régions, lacs et rivières sont moins longtemps gelés, l'océan se réchauffe en surface, le niveau de la mer a augmenté d'une dizaine de centimètres au cours du $\mathrm{XX}^{\mathrm{e}}$ siècle, il y a plus de vapeur d'eau dans l'atmosphère... Malgré quelques zones d'ombre, le consensus est général : le climat se réchauffe.

\section{Le réchauffement du $\mathrm{XX}^{\mathrm{e}}$ siècle : impact de l'homme?}

La troisième conclusion concerne un point, certes important pour le scientifique, mais qui s'avère être la question clé pour le décideur : le réchauffement que nous vivons à l'échelle de la planète a-t-il un lien avec l'augmentation démontrée de l'effet de serre ? Sans évoquer les grandes périodes glaciaires, il suffit de se tourner vers le passé récent pour observer qu'il n'est nul besoin que l'homme intervienne pour que le climat change de façon tout aussi notable qu'il l'a fait au cours du XX siècle. Pour la période que nous appelons le Petit âge glaciaire, entre le milieu du $\mathrm{XV}^{\mathrm{e}}$ siècle et la fin du XIX ${ }^{\mathrm{e}}$ siècle, le constat est sans appel. Il s'appuie sur de nombreux témoignages, tel celui de l'avancée des glaciers alpins ou ceux des peintres flamands et de leurs tableaux d'hivers rigoureux aux fleuves et aux rivières pris par les glaces. Lors de cette période froide qui a atteint son paroxysme entre 1550 et 1700 , la température était, tout au moins en Europe de l'Ouest, d'au moins un degré plus basse que pendant notre $\mathrm{XX}^{\mathrm{e}}$ siècle. À l'inverse, le début du dernier millénaire, époque à laquelle le sud du Groenland était une terre hospitalière, était relativement plus chaud. Difficile dans ces conditions d'affirmer que le réchauffe- ment que nous vivons actuellement est bien lié à l'augmentation de l'effet de serre due aux activités humaines. L'effet de serre augmente et le climat se réchauffe, mais y a-t-il bien relation de cause à effet ? À cette question, le premier rapport du Giec répond que « l'importance du réchauffement observé est grossièrement cohérente avec les prédictions des modèles climatiques, mais elle est aussi comparable à la variabilité naturelle du climat. Le réchauffement observé pourrait donc être dû à cette variabilité naturelle ». Il y a une dizaine d'années, au moment du Sommet de Rio, la réponse des scientifiques était donc «on ne sait pas ». Mais elle a fortement évolué grâce à un ensemble de résultats scientifiques nouveaux, qui permettent aux experts du Giec d'indiquer dans le rapport 1995 « qu'un faisceau d'éléments suggère une influence perceptible de l'homme sur le climat global».

Un premier obstacle est levé. Jusqu'alors, si l'on tient compte de l'augmentation des gaz à effet de serre depuis le début de l'ère industrielle, les modèles d'évolution du climat prévoient un réchauffement voisin de $1{ }^{\circ} \mathrm{C}$. C'est le double de celui observé, estimé alors à une valeur comprise entre 0,3 et $0,6^{\circ} \mathrm{C}$. Mais ces prévisions ne tiennent pas compte de tous les aspects liés à l'activité humaine. Ainsi, elles ignorent l'effet de refroidissement résultant de la présence de particules microscopiques en suspension dans l'air, les aérosols. Ceuxci, produits, en particulier, à partir de composés soufrés résultant partiellement de l'utilisation des combustibles fossiles, rendent l'atmosphère un peu moins transparente et réfléchissent une faible partie de l'énergie qui nous vient du Soleil, de l'ordre de $0,5 \mathrm{~W} \cdot \mathrm{m}^{-2}$. C'est en fait suffisant pour contrecarrer une partie du réchauffement dû à l'augmentation de l'effet de serre et gommer le désaccord entre modèles et observations.

Plus convaincante pour le spécialiste est la mise en évidence de tout une série d'indices qui correspondent à autant d'empreintes attestant que le réchauffement observé n'est vraisemblablement pas uniquement d'origine naturelle. Ces indices s'appuient sur des comparaisons géographiques, saisonnières et verticales du réchauffement, dont les caractéristiques simulées et observées concordent d'autant mieux que les modèles tiennent compte du rôle de l'effet de serre et de celui des aérosols, et non seulement de causes naturelles de variabilité climatique comme les éruptions volcaniques, qui peuvent provoquer un refroidissement notable mais de courte durée, ou 
comme les faibles fluctuations de l'activité solaire. En outre, la prise en compte des aérosols soufrés donne une explication plausible au fait que la température se réchauffe plus la nuit que le jour, car leur effet de refroidissement n'intervient que sur la partie visible du rayonnement, et donc que le jour. Aucun de ces éléments, pris individuellement, ne constitue en soi une preuve, mais c'est leur convergence qui a amené les scientifiques à suggérer que l'action de l'homme était déjà perceptible. C'est une conclusion importante qui, à l'évidence, a joué un rôle clé dans les négociations du protocole de Kyoto. Suggérer, même avec prudence, que les activités humaines commencent à avoir une influence sur le climat met désormais le changement climatique au centre des problèmes que notre société aura à affronter en matière d'environnement et lui donne une dimension socio-économique indéniable.

Le diagnostic s'est affiné entre les rapports du Giec de 1996 et de 2001. Tout d'abord, le climat a continué à se réchauffer et à cela s'ajoute une meilleure connaissance des variations du climat au cours du dernier siècle. Déjà amorcée en 1995, cette avancée se concrétise par la publication d'une courbe de la variation du climat au cours du dernier millénaire, due aux efforts conjugués de paléoclimatologistes qui ont reconstruit différentes séries climatiques à partir d'approches complémentaires et de statisticiens qui les ont combinées et en ont extrait une valeur moyenne. Celle-ci reste entachée d'une grande incertitude (figure 2), mais elle laisse peu de doute : le réchauffement récent sort de la variabilité naturelle. Les modèles climatiques, qui ont fait beaucoup de progrès, confirment ce diagnostic. Des simulations longues montrent que le réchauffement des cent dernières années ne peut vraisemblablement pas être dû uniquement à des causes naturelles. En particulier, le réchauffement marqué des cinquante dernières années ne peut être expliqué que si l'on tient compte de l'augmentation de l'effet de serre. D'où cette conclusion : « Il y a de nouvelles et fortes indications qu'une grande part du réchauffement observé au cours des cinquante dernières années est attribuable aux activités humaines. » De « peut-être » en 1995, nous voici à « probablement » en 2001. Le camp des sceptiques de l'effet de serre se rétrécit et cette conclusion, approuvée par le groupe I au moment de la conférence de La Haye et entérinée dans le rapport de synthèse avant celle de Marrakech, a eu pour conséquence de placer le débat scientifique au second plan... Dans l'esprit des

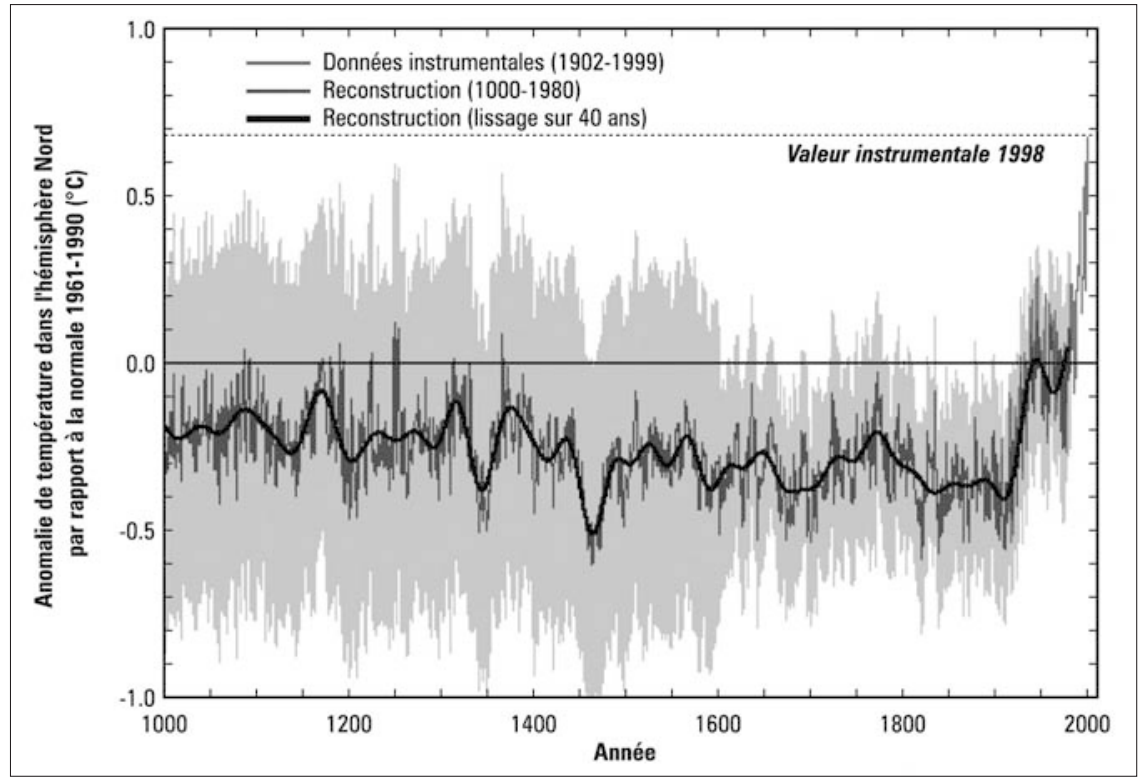

Figure 2 - Estimation de la température moyenne de l'hémisphère nord au cours du dernier millénaire. Cette figure associe l'estimation déduite de la combinaison de différents indicateurs (informations déduites de l'analyse de cernes d'arbres, de coraux, de carottes de glace et d'archives historiques) et des mesures thermométriques pour la partie la plus récente. La partie en grisé indique l'estimation de l'incertitude attachée aux estimations indirectes de la température. (Adaptée du rapport IPCC 2001, [www.grida.no/climate/ipcc tar/wg1/index.htm]) décideurs, l'interrogation puis le doute vis-à-vis de l'action de l'homme sur le climat se sont transformés en quasicertitude.

\section{Des interrogations qui subsistent}

De notre point de vue, des interrogations subsistent, comme celles liées à l'influence éventuelle des changements de l'activité solaire ; il nous faudra au moins une dizaine d'années pour transformer ce « probablement» en certitude. Mais un point extrêmement important est que la réponse à cette question ne préjuge pas de l'ampleur du réchauffement qui nous attend au cours de ce $\mathrm{XXI}^{\mathrm{e}}$ siècle et audelà. Même si l'influence de l'effet de serre additionnel était toujours masquée par la variabilité naturelle du climat, l'augmentation prévue est d'une telle ampleur (de 4 à 8 W.m.-2 supplémentaires) qu'un réchauffement significatif peut sans ambiguité être prévu d'ici à la fin de ce siècle. C'est là la quatrième conclusion du rapport du Giec, qui indique également que de nombreuses incertitudes restent associées à la prévision de l'évolution future de notre climat. Avant de les présenter, nous revenons, plus en détail, sur la façon dont l'homme modifie et continuera à modifier la composition de l'atmosphère.

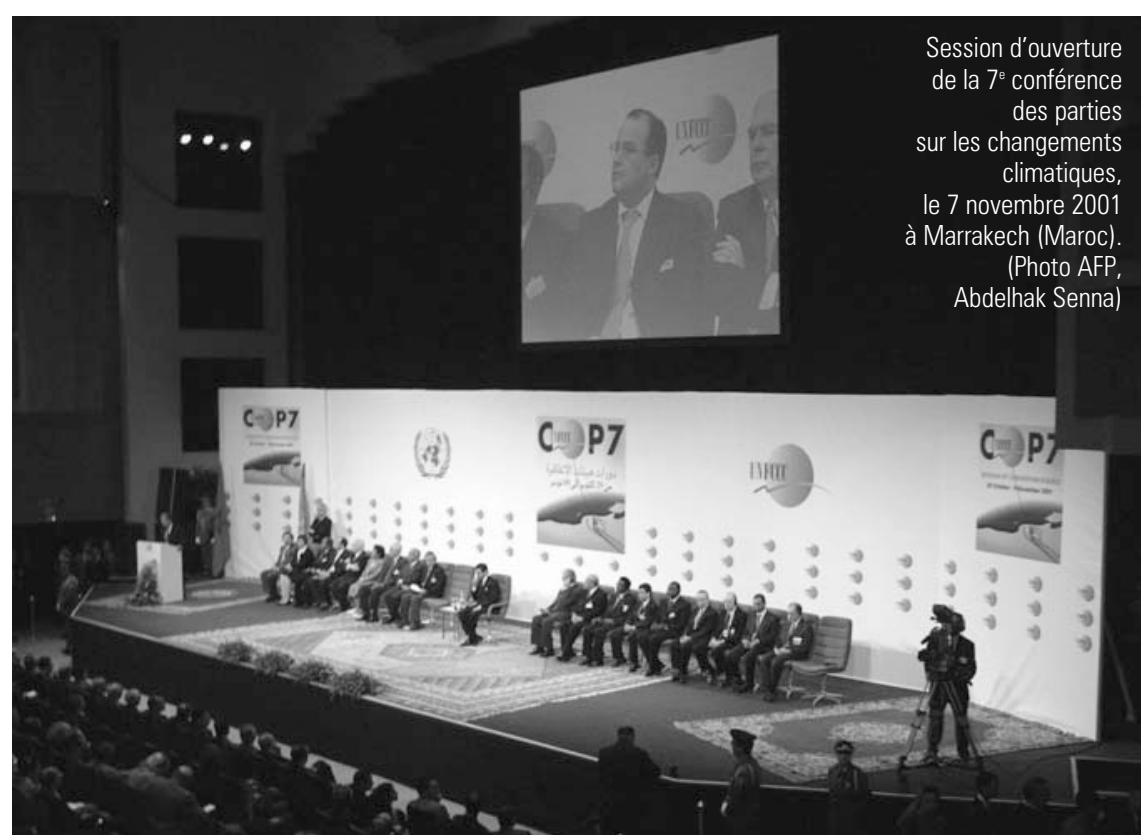




\section{Effet de serre et activités humaines : état des lieux et évolution future}

Sans vouloir négliger l'importance des autres gaz à effet de serre, nous centrerons la discussion sur le dioxyde de carbone, non seulement parce que sa contribution à l'effet de serre additionnel est de loin la plus importante (60\%), mais aussi à cause de son temps de résidence très long dans l'atmosphère. Cela implique que la mâ̂trise de l'évolution de cet effet de serre additionnel passe obligatoirement par un contrôle des émissions de dioxyde de carbone. Cette maîtrise est inscrite dans la CCNUCC, qui stipule que «l'objectif est de stabiliser les concentrations de gaz à effet de serre dans l'atmosphère à un niveau qui empêche toute perturbation anthropique dangereuse du système climatique. Il conviendra d'atteindre ce niveau dans un délai convenable pour que les écosystèmes puissent s'adapter naturellement aux changements, que la production alimentaire ne soit pas menacée et que le développement économique puisse se poursuivre d'une manière durable ».

\section{Le $\mathrm{CO}_{2}$ atmosphérique, partie intégrante du cycle du carbone}

L'augmentation de la teneur en dioxyde de carbone dans l'atmosphère n'est que le reflet d'une perturbation beaucoup plus

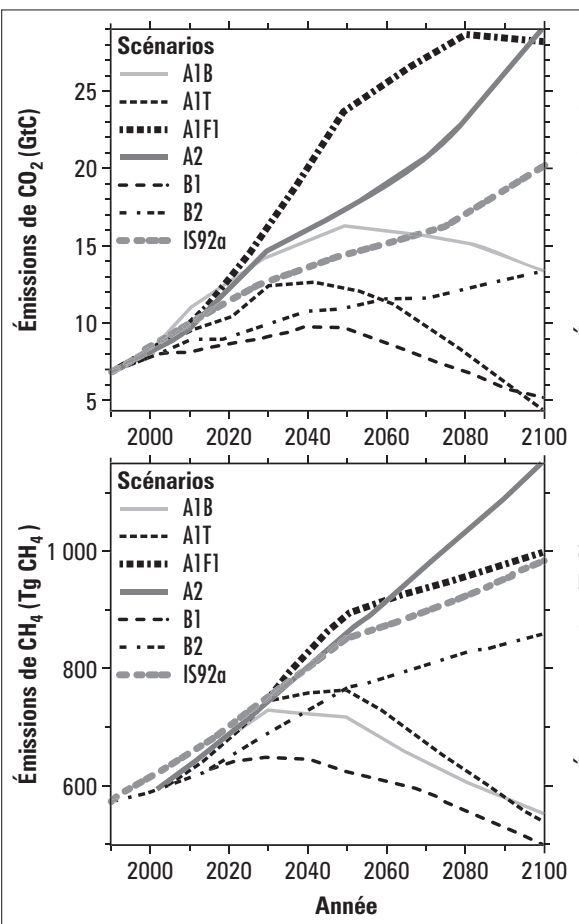

importante, qui affecte l'ensemble du fonctionnement de la planète Terre. Le carbone est en effet contenu dans chacun des compartiments de l'environnement terrestre : atmosphère, océan, biosphère, Terre interne. Dans l'atmosphère, on le trouve essentiellement sous forme de dioxyde de carbone. Sur les continents, on le trouve en profondeur dans les combustibles fossiles et en surface dans la végétation et la matière organique des sols. Dans les océans, il se trouve principalement sous forme de carbonates, en particulier dans le principal réservoir de carbone que constitue l'océan profond. Tous ces compartiments sont en équilibre entre eux grâce à des flux d'échanges permanents, comme la respiration et la photosynthèse entre l'atmosphère et la biosphère, les échanges gazeux entre l'atmosphère et les océans, ou encore l'assimilation du carbone par les micro-organismes dans l'océan. Ce système complexe est équilibré à l'échelle des millénaires. Naturellement, lors de la transition entre une ère glaciaire et une période interglaciaire, les échanges entre compartiments de l'environnement terrestre se modifient et le système ne retrouve son équilibre qu'au bout de quelques siècles, voire quelques millénaires.

Aujourd'hui, l'homme induit à son tour un tel déséquilibre par les sources d'émission additionnelles qu'il contribue à créer : combustion des carburants fossiles (charbon, pétrole, gaz naturel), émissions dues aux pratiques agricoles, déforestation qui diminue la capacité de la végétation à piéger le dioxyde de carbone contenu dans l'atmosphère. Certes, cette perturbation due aux activités humaines reste relativement faible en termes de flux d'échange,
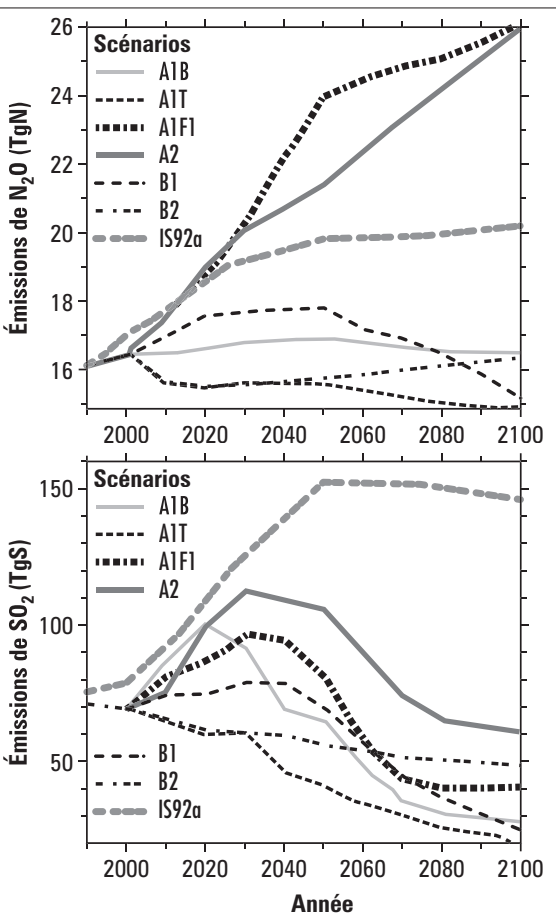

puisqu'elle correspond à l'émission annuelle de 7 milliards de tonnes de carbone (GtC) vers l'atmosphère, dont la plus grande part $(6 \mathrm{GtC})$ est liée à la combustion des carburants fossiles. En effet, les flux d'échange naturels se chiffrent à l'équilibre en dizaines de milliards de tonnes. Mais cette amplitude est suffisante pour modifier les concentrations atmosphériques et, surtout, la rapidité de cette perturbation est inquiétante. Même si une partie (2,0 \pm 0,8 GtC) est absorbée par les océans, une autre $(1,9 \pm 1,9 \mathrm{GtC})$ par la végétation et les sols, ce sont en moyenne plus de $3 \mathrm{GtC}$ qui, chaque année, s'accumulent dans l'atmosphère.

\section{Les émissions de $\mathrm{CO}_{2}$ dans le futur: scénarios extrêmes}

Qu'en est-il pour le futur ? Pour ce qui concerne les émissions, nous nous tournons vers les économistes auxquels le Giec a demandé de proposer différents scénarios prenant en compte les émissions de l'ensemble des gaz à effet de serre, mais également celles des composés soufrés dont, nous l'avons vu, l'effet radiatif est négatif. Ces scénarios, au nombre de 40 , sont établis en tenant compte de différentes possibilités vis-àvis des développements démographiques et économiques futurs et des avancées technologiques qui peuvent raisonnablement être attendues. Nous ne les décrirons pas en détail, mais - et ce n'est pas une surprise - le scénario auquel sont associées les émissions les plus élevées allie un développement économique rapide et une utilisation privilégiée des combustibles fossiles, tandis que les émissions les plus faibles correspondent à une économie de services et d'information avec une recherche optimale de techniques propres et d'efficacité énergétique. Dans le scénario maximal (figure 3 ), les émissions annuelles de dioxyde de carbone vont vers un quadruplement (un peu moins de $30 \mathrm{GtC}$ ) de leur valeur actuelle $(7 \mathrm{GtC})$ à la fin du siècle, tandis que dans le scénario minimal, elles croissent légèrement puis reviennent à un niveau de $5 \mathrm{GtC}$. Les émissions des autres gaz - méthane et protoxyde d'azote - sont également plus importantes dans le scénario maximal, tandis que celles des composés soufrés diminuent dans presque tous les scénarios, du

Figure 3 - Émissions de dioxyde de carbone, de méthane, de protoxyde d'azote et de dioxyde de soufre, dans différents scénarios récemment proposés par le Giec pour la période 1990-2100. [www.grida.no/climate/ipcc_tar/wg1/index.htm] 
fait des efforts importants entrepris depuis plusieurs années pour diminuer les causes de cette pollution.

\section{Les concentrations atmosphériques de $\mathrm{CO}_{2}$ dans le futur : scénarios extrêmes}

L'étape suivante consiste à passer des émissions aux concentrations dans l'atmosphère à partir de modèles biogéochimiques. Pour le dioxyde de carbone, ceux-ci tiennent compte des puits océanique et biosphérique et de leur évolution, ainsi que du fait qu'une fois dans l'océan, le carbone y reste de l'ordre d'un millier d'années tandis que le stockage par la végétation n'est que très transitoire (quelques dizaines d'années). Là non plus, pas de surprise : plus les émissions sont importantes, plus les concentrations deviennent élevées. Le scénario maximal nous entraînerait d'ici à la fin du XXI ${ }^{e}$ siècle vers des concentrations proches de 1000 ppm pour le dioxyde de carbone (triplement), de 3 ppm pour le méthane (doublement) et de 0,45 ppm pour le protoxyde d'azote $(+50 \%)$. Traduction en termes de forçage radiatif : celui-ci serait augmenté de $8 \mathrm{~W} . \mathrm{m}^{-2}$... Mais même le scénario minimal, pour lequel les émissions moyennes de dioxyde de carbone sont proches au cours du XXI ${ }^{\mathrm{e}}$ siècle de leur niveau actuel, a des conséquences alarmantes avec, en 2100, une concentration en $\mathrm{CO}_{2}$ supérieure à 500 ppm et, tenant compte de l'ensemble des composés, un forçage radiatif supplémentaire de près de $3 \mathrm{~W} \cdot \mathrm{m}^{-2}$.

\section{La stabilisation de la concentration atmosphérique de $\mathrm{CO}_{2}$}

Maintenir les émissions de dioxyde de carbone à un niveau constant ne suffit donc pas pour que sa concentration se stabilise, alors que la stabilisation des émissions de gaz à courte durée de vie tels que le méthane se répercute en quelques décennies au niveau de leur concentration dans l'atmosphère. Nous touchons là un point essentiel qui explique pourquoi les scientifiques considèrent que le protocole de Kyoto, qui stipule une légère diminution des émissions de dioxyde de carbone, n'est qu'une étape, d'autant plus qu'en sont dispensés les pays en voie de développement. Cela étant, et nous y revenons plus en détail à la fin de cet article, c'est un premier pas indispensable, un passage obligé pour qu'ultérieurement puissent être mises en place des règles plus contraignantes. Et même si les règles édictées à Kyoto ont été assouplies au cours des conférences des parties successives, même si les États-Unis, principal émetteur de dioxyde de carbone, refusent désormais toute contrainte, l'accord entériné à Marrakech représente toujours un bel espoir.

\section{L'objectif de stabilisation}

Mais beaucoup reste à faire pour que l'objectif de stabilisation visé par la CCNUCC puisse un jour être atteint. Pour nous en convaincre, adressons-nous, non pas aux économistes, mais aux spécialistes du cycle du carbone. Pour que la concentration de dioxyde de carbone se stabilise, il faut que les émissions soient contrebalancées par les puits océanique et biosphérique. Ce que confirment les modèles. Quelle que soit la concentration visée, il faut qu'à un moment donné, dans le futur, les émissions annuelles redescendent en dessous de leur valeur actuelle pour atteindre des valeurs de 2 à $3 \mathrm{GtC}$, voire moins. Si l'on se contente d'une stabilisation à 1000 ppm, ce qui du point de vue du climatologue est complètement déraisonnable, il faudra néanmoins que les émissions n'excèdent jamais 15 $\mathrm{GtC}$, puis reviennent en dessous de leur niveau actuel dans deux siècles. Une stabilisation à 550 ppm, soit déjà le double de la concentration préindustrielle, requiert que les émissions ne dépassent pas $12 \mathrm{GtC}$ en 2040, puis redescendent à leur valeur actuelle vers 2100 et à environ $2 \mathrm{GtC}$ à la fin du XXII siècle. Mais si nous nous assignons un objectif, plus raisonnable, de 450 ppm, c'est dès 2020 que les émissions doivent diminuer pour atteindre leur valeur actuelle en 2050 et moins de $3 \mathrm{GtC}$ à la fin du siècle. Certes, la photosynthèse est favorisée par l'augmentation de la concentration en dioxyde de carbone avec, à la clé, l'espoir d'un puits biosphérique de plus en plus important. Mais d'autres effets (augmentation de la décomposition de la matière organique des sols, répartition différente des espèces...) annihilent l'absorption supplémentaire résultant d'une croissance plus importante de la végétation. Ils vont même au-delà et l'on peut craindre que la végétation ne se transforme de puits de dioxyde de carbone en source... La capacité cumulée d'absorption de l'océan et de la biosphère risque en fait de diminuer à partir du milieu du XXI ${ }^{e}$ siècle. Entre le scénario maximal, celui dans lequel aucun effort n'est fait pour limiter l'effet de serre, et celui certainement souhaitable d'une stabilisation à une concentration pas trop éloignée de sa valeur actuelle, c'est donc à terme un effort de l'ordre d'un facteur 10 qu'il faut réaliser. Le fossé est immense...

\section{Quelques valeurs comparatives}

Aujourd'hui, l'essentiel des émissions de dioxyde de carbone provient de l'utilisation des combustibles fossiles, qui correspond à environ 6 milliards de tonnes de carbone émises par an. La stabilisation des concentrations atmosphériques impliquerait une réduction des émissions de plus de $40 \%$. D' où l'ampleur du problème, puisque ce sont directement les modes de production de l'énergie qui sont ainsi mis en cause. Ceux-ci reposent en effet pour $40 \%$ sur le pétrole, $20 \%$ sur le gaz et $25 \%$ sur le charbon. Il est intéressant ici de réfléchir à ce que pourrait être une répartition équitable des 2 à 3 milliards de tonnes auxquelles il faudrait revenir pour stabiliser la concentration atmosphérique en dioxyde de carbone. Pour une population de 6 milliards d'habitants, chaque habitant de la Terre disposerait donc de 500 kilogrammes de carbone fossile par an. Ce chiffre représente environ $10 \%$ des émissions actuelles d'un Américain, $15 \%$ de celles d'un Allemand, $25 \%$ de celles d'un Français, mais $120 \%$ de celles d'un Indien et $200 \%$ de celles d'un habitant de certains pays d'Afrique subsaharienne. On peut également noter que ce plafond est atteint par un seul aller et retour Paris-New York en avion ou par l'utilisation de 2 tonnes de béton. L'effort à fournir pour stabiliser les concentrations de gaz à effet de serre est donc immense et, en tout cas, incompatible avec une vision du développement fondée sur l'idée de consommer toujours plus. Face aux inégalités actuelles et à la nécessité de développement des pays émergents, la voie de sortie consiste vraisemblablement à faire en sorte que l'Inde et la Chine puissent se développer sans augmenter dans le futur leurs émissions de gaz à effet de serre, mais que, dans le même temps, les pays riches en émettent considérablement moins.

\section{Notre action sur les quinze prochaines années : un engagement sur le $\mathrm{XXI}^{\mathrm{e}}$ siècle}

D'autant, nous l'avons vu, que le problème n'est pas seulement quantitatif et que la variable temps joue aussi sur le niveau de stabilisation, compte tenu notamment des constantes de temps mises en jeu, dans le cas du dioxyde de carbone, par les échanges avec les océans et la biosphère. Ainsi, les décisions que l'on prendra au cours des quinze ou vingt prochaines années fixeront les trajectoires, raisonnables ou 
plus catastrophiques, à l'échelle du XXI siècle et même des siècles suivants.

\section{Quel futur pour les climats de la Terre?}

\section{Une fourchette pour le réchauffement futur}

Alors que l'étude des climats actuel et passé combine observations et simulations numériques, la prévision de son évolution future ne peut faire appel qu'à la modélisation. Prenant en compte les différents scénarios évoqués ci-dessus, les modèles climatiques fixent une fourchette d'augmentation moyenne de la température à l'horizon 2100 comprise entre $1,4{ }^{\circ} \mathrm{C}$ et $5,8^{\circ} \mathrm{C}$ (figure 4). Ainsi, le XXI ${ }^{\mathrm{e}}$ siècle sera certainement un siècle de rupture, caractérisé par une transition extrêmement rapide et une amplification importante du réchauffement moyen comparé à celui observé au $\mathrm{XX}^{\mathrm{e}}$ siècle. L'amplitude de la fourchette tient à deux causes principales, dont chacune représente à peu près la moitié de l'incertitude. La première est bien évidemment notre connaissance imparfaite du système et l'imprécision relative des modèles déjà mise en évidence. La seconde est liée à la difficulté de prévoir nos comportements en matière d'émissions de gaz à effet de serre. Ce réchauffement sera accompagné d'une augmentation du niveau de la mer, largement liée à la dilatation de l'océan et dont l'estimation est comprise entre 9 et $88 \mathrm{~cm}$.

\section{L'importance de l'inertie du système Terre}

Un aspect que met en exergue le troisième rapport du Giec concerne l'inertie du système (figure 5). Nous l'avons discuté pour ce qui concerne le lien entre concentration atmosphérique et émissions de dioxyde de carbone. Il intervient également pour la température et, encore plus, pour le niveau de la mer. Plaçonsnous dans le futur à un moment où les concentrations auront été stabilisées. La température moyenne de la planète va cependant continuer à augmenter avec, suivant les cas, de 50 à $90 \%$ du réchauffement réalisé, et cela à cause de l'inertie de l'océan de surface qui met du temps à s'équilibrer avec l'atmosphère. Suivant le niveau de stabilisation de l'effet de serre, la température pourrait avoir augmenté de 3 à $8{ }^{\circ} \mathrm{C}$ une fois l'équilibre

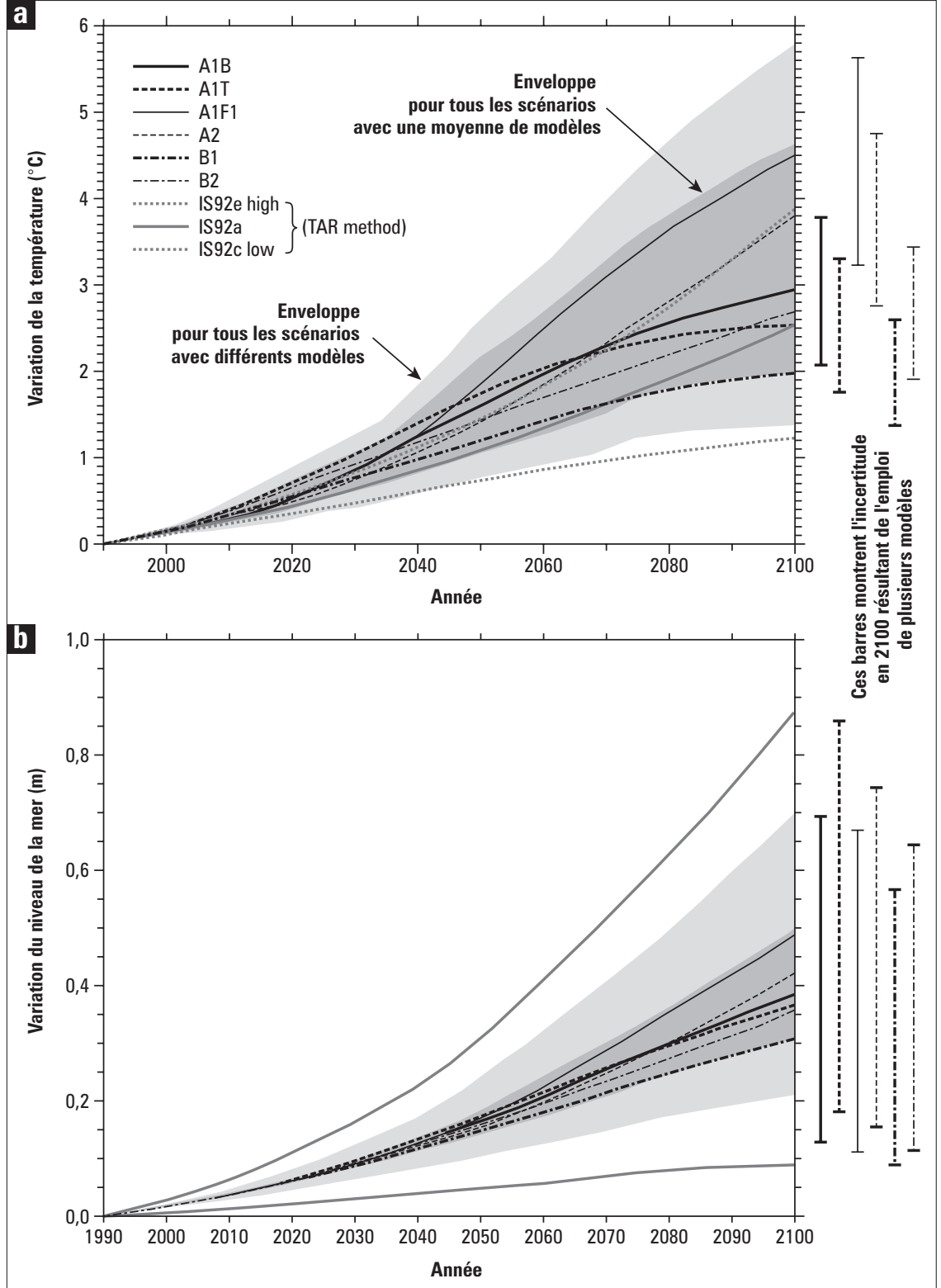

Figure 4 - Prévision, jusqu'en 2100, de l'augmentation de la température moyenne de la planète (a) et du niveau de la mer (b) pour différents scénarios proposés par le Giec. (Adaptée du rapport IPCC 2001, [www.grida.no/ climate/ipcc tar/wg1/index.htm])

atteint. Pour le niveau de la mer, l'inertie est beaucoup plus grande, car c'est l'océan dans son ensemble qui est impliqué dans le processus de dilatation thermique. Une fois les concentrations en dioxyde de carbone stabilisées, le niveau de la mer continuera à monter pendant de nombreux siècles : l'élévation pourrait atteindre 2 mètres au milieu du millénaire dans le cas d'une stabilisation du $\mathrm{CO}_{2}$ à une valeur quadruple de la concentration préindustrielle. S'y ajoute le risque non négligeable que la calotte du Groenland, située dans une région de haute latitude dans laquelle le réchauffement est amplifié, commence à fondre et contribue de quelques mètres supplémentaires à l'élévation du niveau des mers. Même dans le cas d'une politique très volontariste conduisant à la stabilisation de l'effet de serre, on peut donc raisonnablement craindre à l'échéance de quelques siècles des augmentations de température de l'ordre de celles qui ont accompagné le passage d'une période glaciaire à une période interglaciaire et une montée du niveau de la mer qui pourrait atteindre 5 mètres. Nul besoin devant ces chiffres d'insister sur l'absolue nécessité d'une politique de réduction des émissions.

\section{La variabilité du système climatique}

De plus, ces indicateurs correspondent à des valeurs moyennes dont l'estimation est largement fondée sur l'utilisation de modèles simples, qui seuls permettent d'examiner l'ensemble des scénarios. Mais, comme tout système subissant une perturbation, le système climatique est 
également soumis à une variabilité accrue autour de ces valeurs moyennes. Celle-ci peut alors se traduire par une augmentation de la fréquence des événements extrêmes : périodes plus chaudes ou plus froides, pluviosité accrue ou sécheresse intense, renforcement des tempêtes. Elle implique également une modification des phénomènes qui régissent la variabilité naturelle du climat aux échelles de temps interannuelles. Ceux-ci ressortent principalement d'interactions entre les océans et l'atmosphère, à base des lois de la physique, de la dynamique et de la chimie, de simuler le comportement des différents compartiments de l'environnement : océan, atmosphère, biosphères terrestre et marine, cryosphère. Ces modèles sont d'autant plus complexes qu'ils doivent, non seulement rendre compte du comportement de chaque compartiment, mais également des couplages qui les lient entre eux. Ils sont bien sûr loin d'être parfaits, et cela pour deux raisons essentielles.

La concentration en $\mathrm{CO}_{2}$, la température et le niveau de la mer continuent à monter longtemps après que les émissions ont été réduites

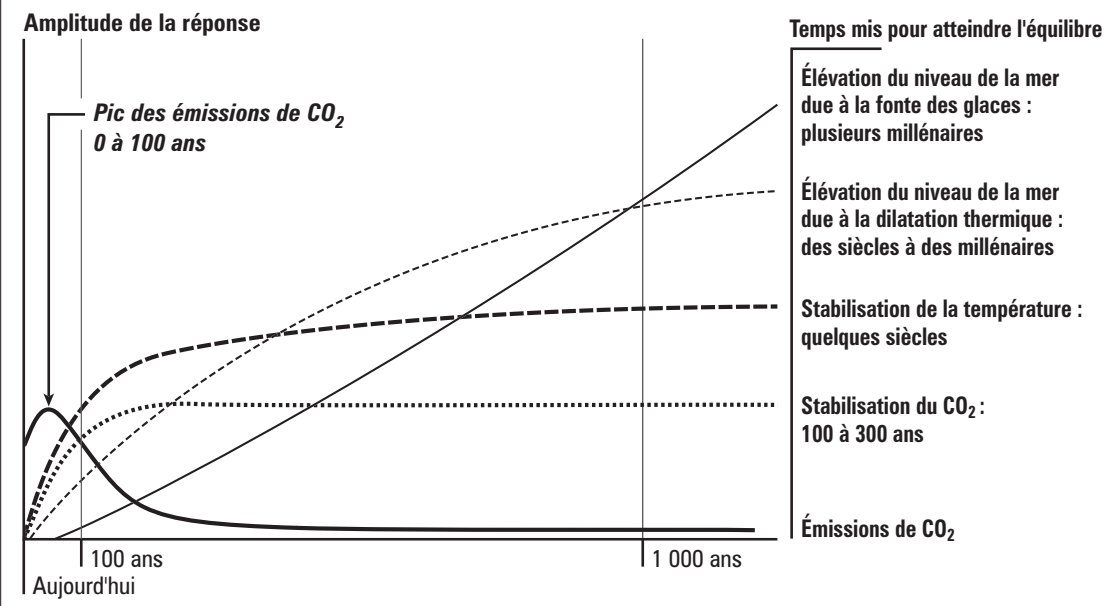

Figure 5 - Cette figure, adaptée du rapport de synthèse du Giec (2001), illustre l'inertie de différentes composantes du système climatique. [www.grida.no/climate/ipcc tar/wg1/index.htm]

l'exemple du phénomène El Niño qui affecte régulièrement, tous les deux à quatre ans, l'océan Pacifique tropical. El Niño est caractérisé par un déplacement des eaux chaudes de surface de l'ouest vers l'est du Pacifique et entraîne une modification importante des régimes de précipitations sur ces régions. Il engendre ainsi des sécheresses redoutables en Australie, en Indonésie et dans le Nordeste brésilien, et des inondations violentes au Pérou, en Argentine et jusqu'en Californie. Les événements El Niño observés récemment semblent montrer que l'intensité de ce phénomène s'accrôit dans un climat plus chaud.

\section{La prévision à l'échelle régionale : les difficultés}

C'est une difficulté supplémentaire que de prévoir ces changements climatiques aux échelles pertinentes des phénomènes, du local au régional. Cela ne peut se faire que grâce à l'outil de modélisation et de simulation du système de l'environnement terrestre qui a été développé au cours des vingt dernières années. C'est un outil extrêmement complexe puisqu'il s'agit, sur la
La première est que notre compréhension du fonctionnement de l'ensemble du système reste incomplète. La seconde tient au fait que les modèles ne peuvent simuler ce fonctionnement avec la résolution quasi infinie qu'impliquerait la prise en compte des échelles les plus fines des phénomènes mis en jeu (résolution spatiale de l'ordre du centimètre ou moins, résolution temporelle meilleure que la seconde). Ils ont aujourd'hui des mailles de l'ordre de quelques dizaines de kilomètres et des échelles de temps de l'ordre quelques dizaines de minutes, voire quelques heures. Cela implique donc que les phénomènes d'échelles plus courtes et plus brèves soient paramétrés, ce qui constitue une importante source d'incertitude. L'une des difficultés essentielles réside dans l'extrême hétérogénéité des échelles spatiales et temporelles, notamment le fait que la différenciation des processus sur les échelles verticales s'opère sur des distances beaucoup plus faibles que sur la dimension horizontale. Une variation d'altitude de quelques dizaines de mètres dans l'océan ou dans l'atmosphère induit des différences extrêmement importantes. Dans ce contexte, une étape importante reste la validation de ces modèles, qui prend en compte l'ensemble des données dont nous pouvons aujourd'hui disposer. Ces validations se fondent aussi bien sur la comparaison avec les observations actuelles que sur la reconstitution des climats du passé.

\section{Les conséquences du changement climatique}

Une difficulté supplémentaire survient lorsque l'on veut prévoir les conséquences potentielles d'un changement climatique. Pour que ces prévisions aient un sens et puissent déboucher sur des mesures d'adaptation ou de correction, celles-ci doivent être évaluées à des échelles d'espace au moins régionales, voire locales. Or, en règle générale, les modèles les plus performants ne s'accordent que sur des fourchettes d'évolution des valeurs moyennes. Leur accord est moins bon dès lors qu'il s'agit de quantifier la variabilité climatique dans le temps et dans l'espace. Pour la température à la surface du globe, les modèles convergent pour montrer que le réchauffement sera plus intense dans les régions de haute latitude, en particulier dans l'hémisphère nord. Mais, lorsque l'on s'intéresse aux précipitations, si tous les modèles prévoient une intensification du cycle de l'eau, avec des précipitations accrues dans les régions de haute et moyenne latitude, et des périodes plus sèches dans les régions tropicales et de basse latitude, la limite entre ces deux régimes reste pour le moins difficile à préciser. Pour rendre compte de cette incertitude, on pourrait dire « qu'il pleuvra plus au nord et moins au sud d'une ligne passant par le centre de la France à 1500 kilomètres près »! Ce qui, on en conviendra, laisse une certaine marge de manœuvre pour l'aménagement du territoire métropolitain.

\section{Conséquences potentielles sur le climat régional}

Néanmoins, un certain nombre de conséquences potentielles du changement climatique peuvent d'ores et déjà être mises en avant, de façon plus ou moins précise. Si la Terre se réchauffe en moyenne, les isothermes vont se déplacer, entraînant des modifications des écosystèmes : mutation des grands types de végétation avec, en particulier, un recul des surfaces boisées, sécheresse accrue dans les latitudes tropicales et risque de conditions extrêmes. En outre, certains écosystèmes assez fragiles seront particulièrement sensibles aux changements climatiques, 
notamment les écosystèmes de montagne et les écosystèmes côtiers. Dans ce dernier cas, l'effet conjugué de variations climatiques locales et de l'élévation du niveau des mers peut avoir des conséquences importantes si l'on se souvient qu'une grande partie de la population du monde vit à proximité des côtes, notamment dans les deltas des grands fleuves. En ce qui concerne la France, il est difficile, compte tenu des incertitudes déjà citées, de prévoir les conséquences à l'échelle régionale d'un changement climatique. Le climat sera vraisemblablement plus sec au sud et plus pluvieux au nord. Les événements extrêmes comme les tempêtes pourraient être plus fréquents et l'enneigement pourrait diminuer sur les reliefs alpins.

\section{L'amplification de la variabilité ?}

Comme nous l'avons déjà noté, tous les modèles montrent qu'en dehors de la valeur moyenne, c'est la variabilité du climat qui risque de s'amplifier au cours des prochaines décennies. Celle-ci dépend pour une large part des couplages entre les différents compartiments du système Terre et est donc d'autant plus difficile à prévoir avec précision. Il est cependant très probable que les régions désertiques et subdésertiques seront particulièrement concernées. Autrement dit, certains des pays en voie de développement, qui doivent déjà faire face à des problèmes difficiles d'alimentation, risquent à nouveau d'être perdants dans la perspective d'un changement climatique. Une autre difficulté tient au fait que, dans cet effort d'appréciation du futur, les données du passé ne sont pas suffisamment précises pour nous apporter des éléments de réponse. Une référence pourrait ainsi être la période de l'optimum climatique de l'holocène, voici environ 8000 ans, quand les températures étaient en moyenne supérieures de 2 à $3{ }^{\circ} \mathrm{C}$ aux valeurs actuelles. La France était alors une gigantesque forêt avec des types de végétation variés. Mais les conditions étaient également trop différentes en termes d'occupation des sols pour que l'on puisse en tirer quelque conséquence que ce soit. Ce que nous confirment en revanche ces reconstitutions du passé, c'est qu'un écart de température de quelques degrés en valeur moyenne suffit à modifier complètement la nature des écosystèmes.

\section{Des surprises possibles}

L'approche des changements climatiques ne peut pas non plus se restreindre à une simple extrapolation linéaire des évolutions actuelles à l'échelle des décennies ou des siècles. Nous ne sommes probablement pas à l'abri d'une « surprise » plus rapide, liée au caractère non linéaire des couplages entre les océans, l'atmosphère et la biosphère. Un tel effet non linéaire s'est déjà produit dans l'atmosphère sous la forme du « trou d'ozone » dans l'Antarctique. Dans le cas du changement climatique, l'exemple d'un ralentissement du Gulf Stream est parfois mis en avant. Le Gulf Stream est ce courant chaud qui amène sur l'Europe de l'Ouest de l'énergie thermique en provenance des zones équatoriales et tropicales et qui nous permet d'avoir un climat largement plus doux que celui de nos amis canadiens, pourtant situés aux mêmes latitudes ${ }^{(1)}$. Il est lié à la plongée des eaux dans les hautes latitudes de l'océan Arctique nord et alimenté par elle. Or, si la fonte des glaces de mer dans cette région, qui semble s'accélérer au cours des dernières décennies, n'a pas d'influence sur le niveau des mers, elle diminue en revanche la salinité de l'océan puisque les glaces de mer sont constituées d'eau douce. Comme la salinité est l'un des facteurs qui détermine précisément la plongée des eaux vers les profondeurs, sa diminution pourrait conduire à un ralentissement du courant. On sait que cette situation d'absence du Gulf Stream s'est déjà produite plusieurs fois dans le passé, à l'échelle de centaines de milliers d'années. En effet, on retrouve dans les sédiments le témoignage de périodes beaucoup plus froides. Dans le cas présent, un tel basculement pourrait s'opérer en quelques décennies et nous obliger à nous adapter rapidement à un climat analogue à celui du Québec. Ainsi, le réchauffement moyen de la Terre risquerait de conduire, du fait d'une instabilité climatique, à un refroidissement rapide de certaines régions. Ce risque n'est cependant pas confirmé par les modèles couplés les plus complexes. En cas de réchauffement climatique, ceux-ci prévoient bien un ralentissement du Gulf Stream lié à l'accroissement de l'évaporation dans les régions tropicales, auquel sont associées des précipitations supplémentaires dans l'Atlantique nord. Cependant, c'est un moindre réchauffement, plutôt qu'un refroidissement, qui est alors prévu sur l'Europe de l'Ouest.

\section{L'impact sur la santé ?}

Un groupe de travail du Giec s'est également intéressé aux conséquences des changements climatiques sur la santé

(1) Ndlr : Le rôle prépondérant du Gulf Stream dans la différence de température entre les deux rives de I'Atlantique nord aux latitudes moyennes a été récemment remis en question (La Recherche, $n^{\circ} 361$, février 2002, p. 40). Cette différence de température proviendrait majoritairement de la circulation générale atmosphérique et de la présence des Rocheuses. humaine. Il importe dès le départ de noter que leur appréciation reste largement de l'ordre du qualitatif. Les premières conséquences pourraient être liées à la recrudescence de phénomènes extrêmes, comme les cyclones, les inondations ou les vagues de chaleur. En revanche, la fréquence des périodes très froides pourrait diminuer à nos latitudes. On peut ensuite penser à des effets secondaires : par exemple, une fréquence accrue des périodes sèches peut entraîner des phénomènes d'érosion, et donc une mise en suspension plus importante de poussières et de pollens dans l'atmosphère, augmentant les risques d'allergie. Des maladies infectieuses comme le paludisme, la fièvre dengue, la fièvre jaune ou les encéphalites risquent de connaître une recrudescence dans les zones de basse et moyenne latitude, en raison de l'augmentation de la température moyenne et de la possibilité de remontée des maladies tropicales vers des régions plus septentrionales. D'autres facteurs peuvent également influencer la santé humaine, comme la diminution du rendement des productions agricoles, en particulier dans les zones tropicales, avec l'occurrence de périodes de sécheresse plus aiguës et des problèmes exacerbés de malnutrition et de famine. Il est enfin pour le moins évident que la vulnérabilité des populations sera directement fonction de leurs ressources naturelles, de leurs ressources techniques et sociales ; on peut donc légitimement penser que ce seront à nouveau les populations les plus fragiles qui auront à subir, en termes d'alimentation et de santé, les conséquences maximales des changements climatiques.

\section{Changement climatique, énergie et développement durable}

\section{La maîtrise raisonnée des émissions}

Il est évident que les climatologues ne peuvent pas « guérir » le climat. Compte tenu des incertitudes actuelles très importantes sur le fonctionnement même du système de l'environnement terrestre, toute tentative de réparation, fondée sur la modification anthropique de tel ou tel processus climatique, s'apparenterait à un jeu d'apprenti sorcier. La seule façon que nous ayons aujourd'hui de diminuer les effets de la perturbation anthropique est d'en limiter l'amplitude. Il nous faut donc réfléchir à 
une maîtrise raisonnée des émissions de gaz à effet de serre, qui renvoie immédiatement au problème des sources d'énergie et du développement durable.

\section{Le protocole de réduction des émissions, Kyoto, 1997}

La prise de conscience de l'ampleur du problème à l'échelle mondiale remonte seulement au début des années 1990 avec la signature de la convention de Rio de Janeiro sur le changement climatique. Un protocole de réduction des émissions a ensuite été signé à Kyoto en 1997, qui prévoit un objectif moyen de réduction des émissions de $5 \%$ en 2008-2012 par rapport au niveau atteint en 1990. Nous sommes effectivement loin de la réduction de $40 \%$ nécessaire pour stabiliser les concentrations de gaz à effet de serre dans l'atmosphère, mais les premières mesures sont souvent les plus difficiles à prendre. De plus, il faut tenir compte du fait que la prolongation des tendances d'accroissement annuel des émissions observées dans les années 1980 conduirait à des émissions en 2010 supérieures de 30 à $40 \%$ à celles de 1990. C'est donc bien une véritable rupture qu'implique le protocole de Kyoto. Ses objectifs sont modulés suivant les différents pays. Les engagements des États-Unis sont ainsi de $-7 \%$, ceux de la Russie de $0 \%$, ceux du Japon de $-6 \%$ et ceux de l'Union européenne de $-8 \%$. Dans la bulle européenne, l'objectif fixé à la France est de $0 \%$, à l'Allemagne de $-21 \%$, au Royaume-Uni de $-12,5 \%$, alors que le Portugal et la Grèce peuvent augmenter leurs émissions de $25 \%$ et l'Espagne de $15 \%$. Les pays en voie de développement, notamment la Chine et l'Inde, ne sont pour l'instant pas concernés par ces réductions.

\section{La position particulière de la France}

La France occupe une position particulière dans ces négociations, liée au fait que la majeure partie de son énergie électrique est issue de modes de production qui ne reposent pas sur l'usage des com- bustibles fossiles : le nucléaire pour $80 \%$ et l'hydroélectrique pour environ $15 \%$. De ce fait, la principale contribution aux émissions de gaz à effet de serre vient des activités agricoles (45 millions de tonnes), dont l'essentiel résulte directement ou indirectement de l'élevage. En effet, en bilan consolidé (Le Treut et Jancovici, 2001), la production d'une tonne de blé revient à émettre 110 kilogrammes d'équivalent carbone, alors que la production d'une tonne de viande correspond à l'émission de 8 tonnes d'équivalent carbone. Les émissions directes des procédés industriels correspondent à la deuxième source d'émissions de gaz à effet de serre (35 millions de tonnes), puis viennent la consommation des ménages (25 millions de tonnes) et les transports ( 25 millions de tonnes). En fait, les émissions de gaz à effet de serre ont diminué en France entre 1970 et 1990, du fait du développement de l'énergie nucléaire, en particulier dans les secteurs déjà cités de la transformation de l'énergie et de l'industrie. Cela explique que notre pays ait, au sein de la «bulle européenne », un objectif affiché de stabilisation des émissions en 2008-2012 par rapport au niveau atteint en 1990. Celui-ci reste néanmoins difficile à atteindre dans la mesure où, les efforts importants ayant déjà été faits dans le domaine des modes de production énergétique et de l'efficacité des processus industriels, la flexibilité principale reste liée à notre capacité à stabiliser les émissions dans les secteurs du transport et de la consommation des ménages, émissions qui ont fortement tendance à augmenter. Ce problème met donc en cause les modes de vie de chacun d'entre nous.

\section{Le protocole de Kyoto : toujours en attente d'être ratifié}

Quatre ans après sa négociation, le protocole de Kyoto n'est toujours pas ratifié, même si les accords entérinés à Bonn, puis à Marrakech en 2001, laissent espérer une ratification prochaine. L'entrée en vigueur du protocole requiert en effet qu'un ensemble de pays représentant plus de $55 \%$ des émissions de gaz à effet de serre s'engagent dans le processus. Le refus des États-Unis de ratifier le protocole de Kyoto impose alors que l'Union européenne, la Russie, le Japon et le Canada ou l'Australie soient parmi les signataires, ce qui semble aujourd'hui acquis. Mais l'attitude des États-Unis, principaux émetteurs de gaz à effet de serre à l'échelle mondiale, pose les conditions d'une réduction rapide des émissions, malgré le volontarisme affiché par les autres pays. Il est plus que probable que les objectifs, même modestes, fixés par le protocole de Kyoto pour la période de référence 2008-2012 ne seront pas atteints.

Aujourd'hui, l'Union européenne n'a réduit ses émissions que de $1 \%$. Quant aux États-Unis, dont l'objectif est de $-7 \%$, ils en sont à $+20 \%$ par rapport à l'année de référence 1990. Seuls les pays de l'Est et la Russie ont d'ores et déjà réussi à remplir leurs objectifs, du fait du ralentissement majeur de leur économie au cours des années 1990, qui se traduit par une baisse des émissions de $38 \%$; ce qui, dans le cadre de la mise en œuvre d'un marché mondial de permis d'émission, excite bien des convoitises. Cette difficulté qu'ont les principaux pays à se conformer aux objectifs du protocole n'est d'ailleurs pas indépendante des positions affichées aujourd'hui quant à sa ratification. En ce qui concerne les pays en développement, les mécanismes mis en œuvre dans le cadre de la convention de Rio de Janeiro et du protocole de Kyoto, notamment ceux liés au développement propre, portent l'espoir d'une solution raisonnable à moyen terme.

La ratification du protocole de Kyoto est certainement une première étape nécessaire, si l'on considère que de notre capacité à prendre rapidement les décisions qui s'imposent dépendra pour une large part l'avenir des générations futures. C'est tout l'enjeu d'un développement durable à l'échelle de la planète, qui ne saurait laisser de côté les pays en voie de développement. Il implique un changement décisif de nos modes de vie et de consommation et une volonté partagée par une large partie de l'humanité. C'est un enjeu politique déterminant pour le $\mathrm{XXI}^{\mathrm{e}}$ siècle.

\section{Bibliographie}

\footnotetext{
: Barbault R., A. Cornet, J. Jouzel, G. Mégie, I. Sachs et J. Weber, 2002 : Johannesburg 2002, Sommet mondial du développement durable. Quels enjeux ? Quelle - contribution des scientifiques? Ministère des Affaires étrangères, Paris, 210 p.

- Le Treut H. et J.-M. Jancovici, 2001 : L'effet de serre : allons-nous changer le climat? Flammarion, collection « Dominos », Paris, 128 p.

- Changements climatiques : de la convention de Rio aux accords de Bonn et Marrakech. Guide explicatif des accords internationaux. [www.effet-de- serre.gouv.fr/main.cfm?page =fr/accords/accords.htm
} 